\title{
Metabolic demand stimulates CREB signaling in the limbic cortex: implication for the induction of hippocampal synaptic plasticity by intrinsic stimulus for survival
}

\author{
Nelly M. Estrada ${ }^{\dagger}$ and Masako Isokawa* \\ Department of Biological Sciences, The University of Texas at Brownsville, Brownsville, TX, USA
}

Edited by:

Federico Bermudez-Rattoni,

Universidad Nacional Autónoma de

México, Mexico

Reviewed by:

Manfred Schedlowski, University of

Duisburg-Essen, Germany

Jorge Medina, Universidad de Buenos

Aires, Argentina

*Correspondence:

Masako Isokawa, Department of

Biological Sciences, The University of

Texas at Brownsville, 80 Fort Brown,

Brownsville, TX 78520, USA

e-mail:masako.isokawa@utb.edu

${ }^{+}$Current address: Nelly M. Estrada

Texas Tech University Health Sciences

Center, Paul L. Foster School of

Medicine, 4800 Alberta Ave, El Paso, TX 79905, USA
Caloric restriction by fasting has been implicated to facilitate synaptic plasticity and promote contextual learning. However, cellular and molecular mechanisms underlying the effect of fasting on memory consolidation are not completely understood. We hypothesized that fasting-induced enhancement of synaptic plasticity was mediated by the increased signaling mediated by CREB (cAMP response element binding protein), an important nuclear protein and the transcription factor that is involved in the consolidation of memories in the hippocampus. In the in vivo rat model of $18 \mathrm{~h}$ fasting, the expression of phosphorylated CREB (pCREB) was examined using anti-phospho-CREB (Ser133) in cardially-perfused and cryo-sectioned rat brain specimens. When compared with control animals, the hippocampus exhibited up to a twofold of increase in pCREB expression in fasted animals. The piriform cortex, the entorhinal cortex, and the cortico-amygdala transitional zone also significantly increased immunoreactivities to pCREB. In contrast, the amygdala did not show any change in the magnitude of pCREB expression in response to fasting. The arcuate nucleus in the medial hypothalamus, which was previously reported to up-regulate CREB phosphorylation during fasting of up to $48 \mathrm{~h}$, was also strongly immunoreactive and provided a positive control in the present study. Our findings demonstrate a metabolic demand not only stimulates cAMP-dependent signaling cascades in the hypothalamus, but also signals to various limbic brain regions including the hippocampus by activating the CREB signaling mechanism. The hippocampus is a primary brain structure for learning and memory. It receives hypothalamic and arcuate projections directly from the fornix. The hippocampus is also situated centrally for functional interactions with other limbic cortexes by establishing reciprocal synaptic connections. We suggest that hippocampal neurons and those in the surrounding limbic cortexes are intimately involved in the metabolism-dependent plasticity, which may be essential and necessary for successful achievement of adaptive appetitive behavior.

Keywords: immunohistochemistry, entorhinal cortex, amygdala, piriform cortex, hypothalamus

\section{INTRODUCTION}

The family of CREB (cAMP response element-binding protein) transcription factors are involved in a variety of biological processes including the development and plasticity of the nervous system (Mayr and Montminy, 2001; Pittenger et al., 2002). In order for CREB to be active, it needs to be phosphorylated before being translocated to the nucleus. Thus, identification of a phosphorylated form of CREB (pCREB) is a reliable assay for predicting the occurrence of functional plasticity, learning, and memory in neurons.

Feeding is a homeostatic process controlled by the hypothalamus. In the hypothalamus, the magnitude of the expression of pCREB changes depending on the metabolic demand (Sheriff et al., 1997). During starvation, the expression of pCREB increases in the orexigenic peptide-producing neurons in the arcuate nucleus (Morikawa et al., 2004; Shimizu-Albergine et al., 2001). The elevated expression of pCREB persists until feeding is initiated and metabolic balance is restored. This suggests that CREB is constitutively active in the arcuate nucleus of the hypothalamus during starvation.
When the metabolic demand increases, animals have to locate food by adopting various food-searching strategies, recalling the location of food, and remembering its availability. Learning the predictive cues for reward and connecting that information with appropriate responses require motivation-driven associative learning and long-term memory, which likely involves the limbic cortexes including the hippocampus. However, cellular mechanisms underlying the functional link between peripheral metabolic signaling and higher neuronal functions such as synaptic plasticity and learning are not well understood in the limbic brain.

The goal of this project is to examine the effect of a metabolic demand (fasting) on the neuron activities in the hippocampus and the surrounding limbic cortexes. We hypothesized that fastinginduced metabolic demand not only initiates constitutive activation of CREB in the hypothalamus but also signals to the limbic cortexes and the hippocampus through hormonal regulation or direct synaptic connections. Such signaling may be detected as increased activities of pCREB during fasting in the limbic brain. Indeed, our findings support the above hypothesis and provide evidence that 
hippocampus and the limbic cortex are intimately involved in the metabolism-dependent plasticity. Such an intimate communication between the feeding center and the memory center in the brain might be essential and necessary for the successful establishment of adaptive appetitive behavior.

\section{MATERIALS AND METHODS IN VIVORAT MODEL OF FASTING}

Rats with the age of 21-24 day old were used in the present study. All protocols were approved by the IACUC in the University of Texas at Brownsville. The rats were fasted for $18 \mathrm{~h}$. During fasting, the animals were housed individually in our animal facilities where control rats were also housed individually. Light-dark cycle remained unchanged during fasting, and water was available ad lib. At the end of the fasting period, the animals were cardially perfused with $4 \%$ paraformaldehyde dissolved in $0.1 \mathrm{M}$ sodium phosphate buffered saline ( $\mathrm{pH}$ 7.2). At the end of the perfusion, the brains were removed and stored in the $4 \%$ paraformaldehyde-containing PBS with 30\% sucrose for cryo-protection.

\section{IMMUNOHISTOCHEMISTRY}

The fixed brains were frozen and sectioned in $40 \mu \mathrm{m}$ thick. The cut brain sections were rinsed with the sodium phosphate buffer and treated with BSA (5\%) and Triton- $100 \times(0.2 \%)$ for $2 \mathrm{~h}$. A primary antibody specific for the phosphorylated form of CREB (antiphospho-CREB, Ser133, raised in the rabbit, Cell Signalling) was added to PBS (1:50) and the brain sections were incubated in a freefloating manner in the primary antibody-containing PBS for $48 \mathrm{~h}$ at $4^{\circ} \mathrm{C}$. Subsequently, the sections were rinsed and incubated with a biotinylated anti-rabbit secondary antibody for $2 \mathrm{~h}$ at room temperature. At the end of the incubation, the sections were processed for the visualization of immunoreactive product using an ABC kit (Vector lab) and diaminobenzidine. Brain specimens taken from control rats and fasted rats were processed simultaneously using the same butch of solutions in order to minimize the variability in the visualization of immunoreactivity and DAB reaction product.

\section{DATA ANALYSIS}

Reaction products were observed using a light microscope. The number and location of pCREB immunoreactive neurons were mapped manually on the rat brain atlas (Paxinos and Watson, 1993) while observing the specimens using a light microscope with $10 \times$ and $40 \times$ objectives. The number of sampled neurons were totaled and divided by the number of sampled locations in a given brain region to obtain the average and the standard error of the mean of pCREB immunoreactive neurons in fasted animals and control animals. In addition to the manual counting of the immunoreactive neurons, the identical pool of the brain sections from fasted animals and control animals were photographed digitally using a CCD camera and Scion Capture software. Optical images $\left(37,000 \mu \mathrm{m}^{2}\right)$ were acquired with a $40 \times$ objective from the CA1, CA3, and dentate gyrus of the hippocampus, the entorhinal cortex, the piriform cortex, the amygdala, the amygdalo-cortical transitional zone, and the arcuate nucleus of the hypothalamus. Individual neurons that expressed pCREB immunoreactivity were selected based on the optical density and cell size (area). They were marked with ROIs. Data were quantified and analyzed using IPLab software (BD Bioscience).
Results were consistent between manual counting and the optical counting. Thus, results obtained by the optical counting are summarized and shown in the present study.

\section{RESULTS \\ FASTING INCREASES THE EXPRESSION OF pCREB IN THE ARCUATE NUCLEUS OF THE HYPOTHALAMUS}

The arcuate nucleus of the hypothalamus has been reported to increase the expression of pCREB in response to fasting for up to $48 \mathrm{~h}$ (Shimizu-Albergine et al., 2001). This increase was unique to the arcuate nucleus and independent of the ventromedial hypothalamus or the dorsomedial hypothalamus. Indeed, in spite of dense local synaptic connections among these subnuclei of the hypothalamus, neither ventromedial nor dorsomedial hypothalamus was reported to show any difference in the pCREB expression between control and fast. In the present study, we sampled pCREB-immunoreactive neurons from the arcuate nucleus in the control rats ( 34 brain sections that contained the arcuate nucles in six rats) and $18 \mathrm{~h}$-fasted rats (38 brain sections that contained the arcuate nucleus in six rats). We observed a significant increase of pCREB expression in the arcuate nucleus in the fasted rats for 18 h (Figure 1). When compared with control rats, the expression of pCREB was fourfold greater in the arcuate nucleus of the fasted rat hypothalamus (Figure 1E). This finding demonstrates that our rat model of $18 \mathrm{~h}$-fasting successfully stimulated the hypothalamic arcuate neurons and activated CREB-mediated signaling in response to an increased metabolic demand.

\section{FASTING INCREASES THE EXPRESSION OF PCREB IN THE HIPPOCAMPUS}

The arcuate nucleus sends direct fiber connections to the hippocampus via the fornix (Conrad and Pfaff, 1976; Shen and Anderson, 1980). Dorsal fornix projects to CA1, CA3, and dentate gyrus (DG) of the hippocampus and to the entorhinal cortex (Swanson, 1980). In return, the CA1 of the hippocampus sends fibers bi-synaptically to the arcuate nucleus via the subiculum through post-commissural fornix (Swanson, 1980). These reports provide anatomical substrate for a tight functional coupling between the hypothalamic arcuate neurons and the hippocampal neurons as well as extrahippocampal limbic cortical neurons. Because we observed a robust increase in pCREB in the arcuate nucleus in the fasted rats, we investigated the expression of pCREB in the hippocampus and the surrounding limbic cortexes in these animals.

pCREB immunoreactive neurons were sampled in the dentate gyrus (DG), CA1 and CA3 of the hippocampus in control rats and fasted rats. One hundred and twenty-eight brain sections were analyzed in six fasted rats, and 93 brain sections were analyzed in six control rats. In control animals, pCREB expression was detected in all three hippocampal subfields. The CA3 exhibited the highest expression of pCREB, which was three times greater than that of the DG and twice greater than that of CA1 (Figure 2).

The levels of pCREB expression in CA1 and DG were similar and also comparable with the level of expression of pCREB observed in the arcuate nucleus of control animals. Varying degrees of pCREB expression in different hippocampal subfields suggest that each hippocampal subfield has a homeostatic level of CREB activity that appears unique to each hippocampal region under a physiological 

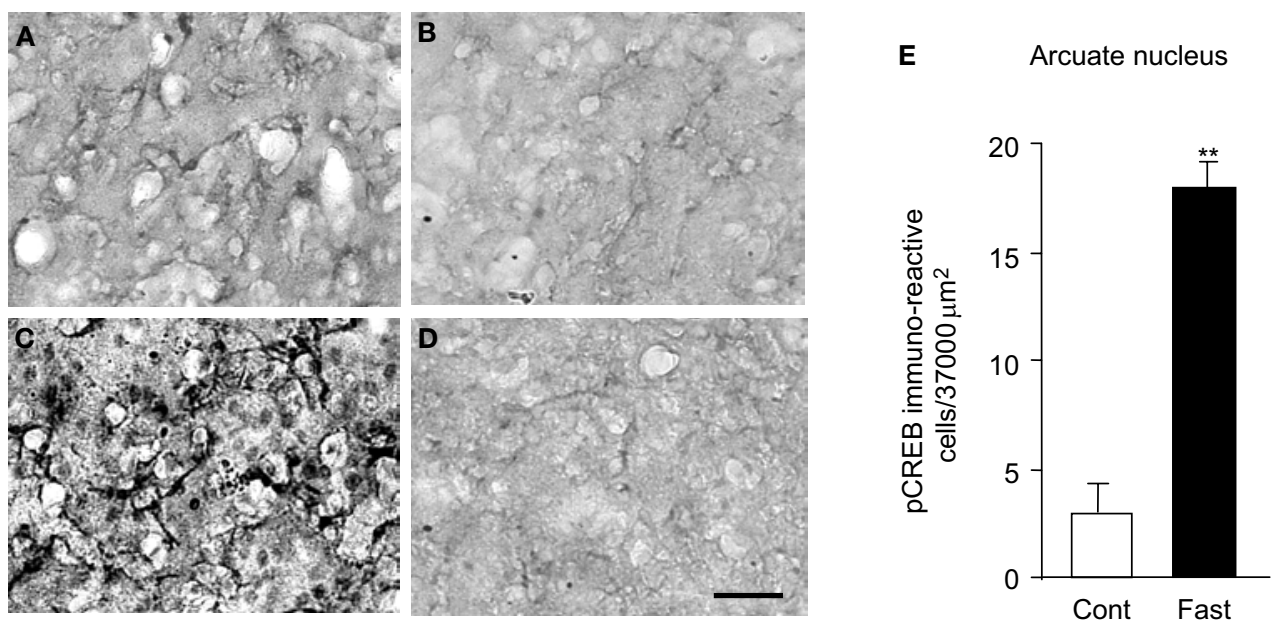

FIGURE 1 | pCREB immunoreactivity in the arcuate nucleus of the hypothalamus in control (A, B) and fasted (C, D) rats. (B) and (D) were processed without a primary antibody. (E) Results were quantified for the mean \pm SEM (standard error of the mean). Calibration: $50 \mu \mathrm{m} .{ }^{*}{ }^{*} p<0.001$.

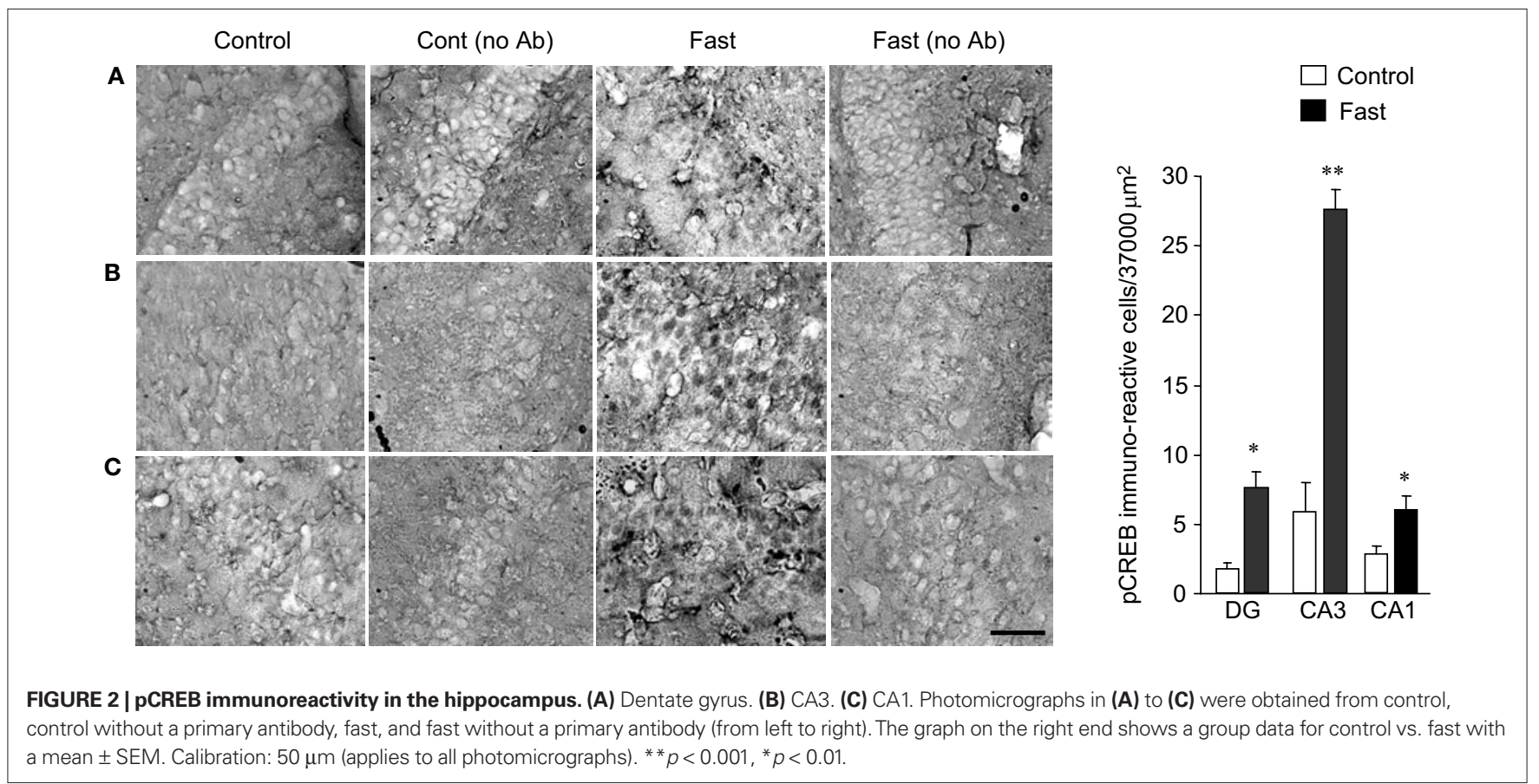

condition. Although we cannot rule out the possibility that experimental methodology such as cardiac perfusion and decapitation could affect the expression level of pCREB (O'Callaghan and Sriram, 2004), our result demonstrated the presence of regionspecific magnitude of CREB activities in different hippocampal subfields as a homeostatic response.

The activity of CREB increased in response to fasting in the three hippocampal subfields of CA1, CA3, and DG (Figure 2). As expected, the total number of neurons that expressed PCREB within a defined unit area was highest in the CA3 (27.3 neurons \pm 2.11 SEM in $37,000 \mu \mathrm{m}^{2}$ ), which was a fourfold of increase compared with the pCREB expression observed in the CA3 of control animals. DG showed a threefold increase in the level of pCREB expression in fasted animals (8.2 neurons \pm 0.92 SEM), and CA1 showed a twofold increase in the level of pCREB expression in fasted animals (6.9 neurons $\pm 2.01 \mathrm{SEM}$ ) when they were compared with control animals. The present finding demonstrates that fasting does have an effect on the hippocampal neuron activities and increases the expression level of pCREB.

\section{FASTING INCREASES THE EXPRESSION OF PCREB IN THE LIMBIC CORTEX}

The hippocampus has intimate anatomical connections with the entorhinal cortex. The medial and lateral entorhinal cortex projects to DG in the hippocampus monosynaptically (Ohshima et al., 2008). In return, the CA1 neurons project back to the entorhinal 
cortex via the subiculum bi-synaptically (Finch et al., 1986a). These connections are functionally excitatory, and thus the hippocampal and entorhinal neurons stimulate their target neurons mutually. In addition, there are reciprocal connections between the entorhinal cortex and the amygdala (Brothers and Finch, 1985; Finch et al., 1986b). The amygdala also projects their neuron fibers to the piriform cortex (Meurisse et al., 2009) as well as to the transition zone between the amygdala and the cortex (Fudge and Tucker, 2009). Based on these anatomical and functional evidence, we sampled pCREB immunoreactive neurons in the entorhinal cortex, piriform cortex, amygdala, and the cortico-amygdala transitional zones.

In control animals, the level of pCREB expression was detected in all four regions, i.e., the entorhinal cortex, piriform cortex, corticoamygdala transitional zone, and the amygdala. The magnitude of pCREB expression was similar among these four regions and averaged around 30 neurons per a defined unit area (Figure 3 ). Surprisingly, in control animals, the level of pCREB expression in all three limbic cotexes and the amygdala was higher than the level of expression detected in the hypothalamus and the hippocampus. As a matter of fact, the magnitude of PCREB expression of 30 neurons per a defined unit area was equivalent of the expression level observed in the CA3 and the arcuate nucleus of the fasted animals. This finding suggests that the intrinsic level of CREB activities in the limbic cortex might be higher than that of the hypothalamus and the hippocampus.

Eighteen hours of fasting stimulated the CREB activities and increased the expression level of pCREB in the entorhinal cortex, piriform cortex, and the amygdalo-cortical transitional zone.

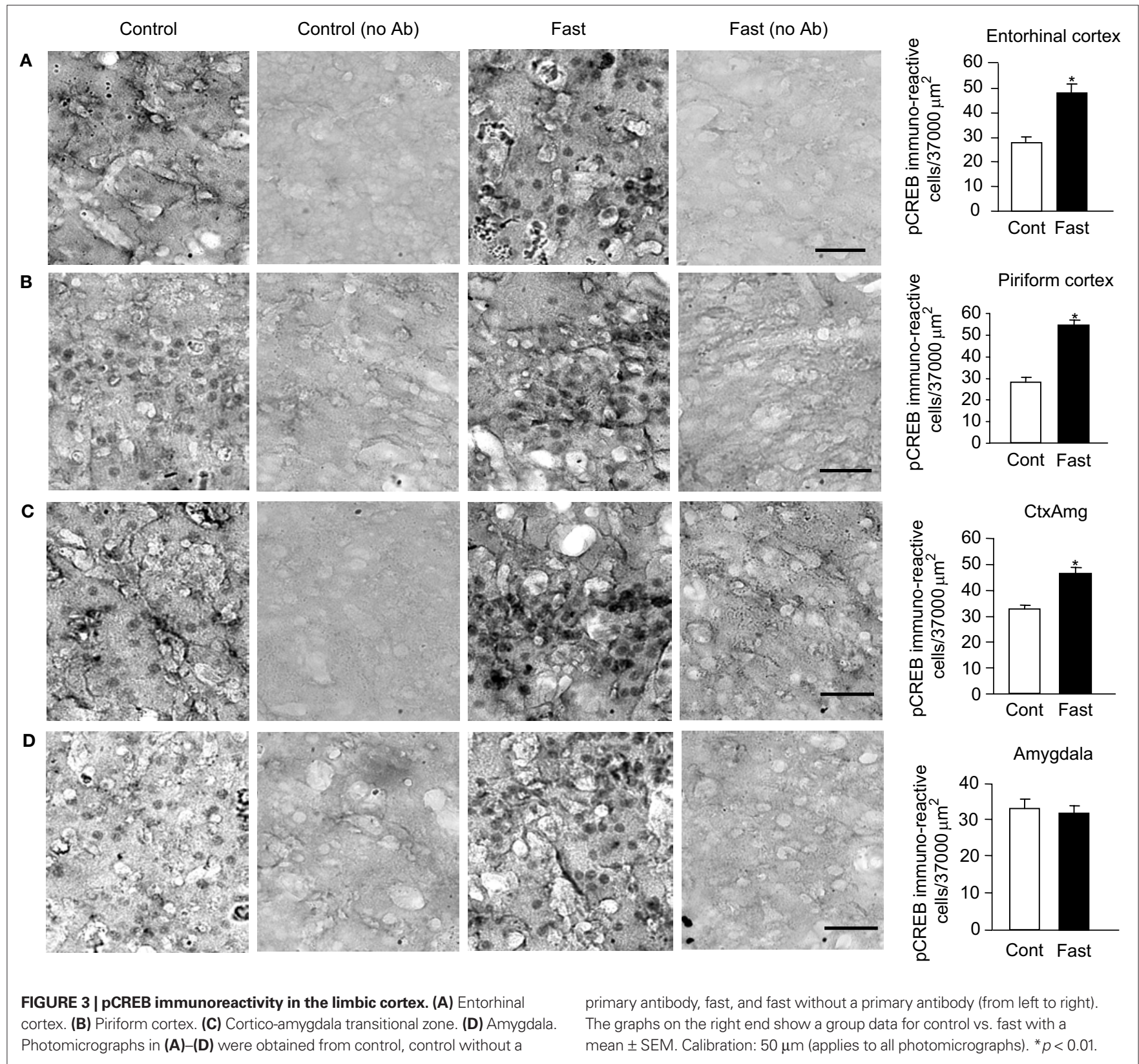


The entorhinal cortex increased pCREB expression by $60 \%$ in response to fasting. The piriform cortex increased pCREB expression by $89 \%$, and the amygdalo-cortical transitional zone increased pCREB expression by $38 \%$. These findings demonstrate that both entorhinal and piriform cortexes do respond to an increased metabolic demand by stimulating CREB activities. On the other hand, the amygdala did not show any increase of pCREB expression in response to fasting (Figure 3). The average number of neurons that were pCREB immunoreactive remained near 30 in control and fast. There must be a cellular mechanism that may block excitatory communication from the limbic cortex to the amygdala. A candidate zone that might be responsible for this blockage could be the transitional zone, as the amygdalo-cortical transitional zone increased the level of pCREB expression by only $38 \%$ in the fasted animals, which was a half of those observed in the entorhinal cortex and the piriform cortex. There is a report to show that the hippocampus sends an inhibitory synaptic connection to the amygdala (Mello et al., 1992). Thus, increased activities of neurons and pCREB in the hippocampus in response to fasting might in turn inhibited the activities of the amygdala neurons leading to the lack of changes in the pCREB expression. These findings suggest that synaptic connections and their functional interactions could play an important role in the expression of pCREB in the hippocampal and limbic cortexes in response to fasting. Of course, we cannot rule out the possibility that metabolic hormones exerted a direct effect on the induction of CREB activities in each brain region independently as we proposed this possibility later in Section 'Discussion'.

\section{DISCUSSION}

The present study demonstrated that (1) major limbic cortexes of the rat brain, the hippocampus, the entorhinal cortex, the piriform cortex, and the amygdalo-cortical zone, responded to an increased metabolic demand ( $18 \mathrm{~h}$ of fasting) by stimulating the activity of CREB thus increasing the expression level of pCREB; (2) the magnitude of increase in the pCREB expression in response to increased metabolic demand varied among the limbic cortexes; and (3) an intrinsic level of CREB activities, measured by the expression level of pCREB in control animals, also varied among different limbic cortexes exhibiting a structure-specific level of CREB homeostasis. Together these data suggest that a homeostatic level of CREB activity and the responsiveness of CREB to increased metabolic demand are unique in each different limbic brain structure.

Our finding of a neuroanatomically differentiated pattern of CREB activation in the limbic brain indicated that there was a varying magnitude of cross talk between brain areas involved in cognitive, motivational, and metabolic-regulatory functions during fasting. This suggests that natural reward such as food may have differential effects on the activation of cAMP signaling pathways in each region of the limbic brain. A similar observation for a region-specific up-regulation of $\mathrm{pCREB}$ expression was reported in response to the administration of antidepressant (Thome et al., 2000).

cAMP cascade is reported to be integrally involved in the cellular adaptations underlying learning and memory in the hippocampus (Abel et al., 1997). The present findings demonstrate that this signaling cascade is apparently involved in the process of procuring sufficient energy. The present findings also suggest the importance for understanding how metabolic need may be translated into neuronal activities that involve cognitive and motivational brain areas. Constitutively active CREB protein was reported to facilitate the late phase of long-term potentiation, the best-characterized candidate mechanisms for many forms of experience-dependent plasticity, learning, and memory (Barco et al., 2002). Thus, the contribution of metabolic demand-induced PCREB expression may be to provide an increased basal level of CREB activity that may act favorably to amplify gene expression induced by a specific form of learning.

Peripheral metabolic demand releases hormones such as ghrelin. Circulating ghrelin was reported to enhance long-term potentiation and spatial learning in the hippocampus (Diano et al., 2006; Moran and Gao, 2006). Fasting facilitated the rate of ghrelin crossing the blood brain barrier (Banks et al., 2008). There is a high level of ghrelin receptor expressed in the hippocampus (Harrold et al., 2008). Thus, ghrelin could be a candidate molecule to activate CREB signaling in the hippocampus during fasting. However, the remaining limbic cortexes do not show an appreciable level of ghrelin receptors even if they expressed a high level of pCREB expression in response to fasting. This suggests that neuronal activities in various limbic regions during fasting may not be explained by single hormonal regulation. Interestingly, we did not observe any change in the expression of pCREB in the amygdala in fasted animals. The amydala does not express a significant level of ghrelin receptor. There is a report to show that ghrelin did not have any effect on food intake when it was injected directly to the amygdala (Carlini et al., 2004). Accordingly, ghrelin may not be the molecule that stimulates the expression of PCREB in the amygdala. However, it should be noted that, when ghrelin was administered intravenously to human subjects, a visual cue for food (food pictures) stimulated the activity of the amygdala (Malik et al., 2008). Visual food cues can trigger hedonic feeding independent of homeostatic feeding. Together these evidence suggest the likely-involvement of complexly-interwoven hormonal and non-hormonal metabolic signals in the CREB activation in various limbic cortexes during fasting. It may be premature to propose any single molecule or hormone that can explain an increased expression of pCREB in response to metabolic demand throughout all limbic regions that we investigated as a neuronal system.

In conclusion, we demonstrated that CREB activation occurred in response to fasting not only in the arcuate nucleus of the hypothalamus but also in several representative limbic cortexes including the hippocampus. The challenge now is to identify the target genes that are influenced by the CREB activation in response to metabolic demand in these different limbic cortexes. Another challenge is to identify the potential molecules that are responsible to activate CREB signaling pathways during fasting in the limbic cortexes including the hippocampus. The result of the present study, in combination with the future approach, should provide a fuller understanding of the role of CREB in the integrative neuronal control in metabolic efficiency.

\section{ACKNOWLEDGMENTS}

This work was supported by the Borderplex Council Award from the University of Texas Health Care System and grants from NIH R15DA021683 and SC1GM081179 (to MI). NME was supported by the American Physiological Society Undergraduate Summer Research Fellowship in 2008. 


\section{REFERENCES}

Abel, T., Nguyen, P. V., Marad, M., Deuel, T. A. S., Kandel, E. R., and Bourtchouladze, R. (1997). Genetic demonstration of a role for PKA in the late phase of LTP and in hippocampusbased long-term memory. Cell 88, 615-626.

Banks, W. A., Burney, B. O., and Robinson, S. M. (2008). Effects of triglycerides, obesity, and starvation on ghrelin transport across the blood-brain barrier. Peptides 29, 2061-2065.

Barco, A., Alarcon, J. M., and Kandel, E. R. (2002). Expression of constitutively active CREB protein facilitates the late phase of long-term potentiation by enhancing synaptic capture. Cell 108, 689-703.

Brothers, L. A., and Finch, D. M. (1985). Physiological evidence for an excitatory pathway from entorhinal cortex to amygdala in the rat. Brain Res. 359, 10-20.

Carlini,V.P.,Varas,M.M.,Cragnolini, A. B., Schiöth, H. B., Scimonelli, T. N., and de Barioglio, S. R. (2004). Differential role of the hippocampus, amygdala, and dorsal raphe nucleus in regulating feeding, memory, and anxietylike behavioral responses to ghrelin. Biochem. Biophys. Res. Commun. 313, 635-641.

Conrad, L. C., and Pfaff, D. W. (1976). Efferents from medial basal forebrain and hypothalamus in the rat. II. An autoradiographic study of the anterior hypothalamus. J. Comp. Neurol. 169, 221-261

Diano, S., Farr, S. A., Benoit, S. C., McNay, W.C., da Silvo, I., Horvath, B., Gaskin, F. S., Nonaka, N., Jaeger, L. B., Banks, W. A., Morley, J. E., Pinto, S., Sherwin, R. S., Xu, L., Yamada, K. A., Sleeman, M. W., Tschöp, M. H., and
Horvath, T. L. (2006). Ghrelin controls hippocampal spine synapse density and memory performance. Nat. Neurosci. 9, 381-388.

Finch, D.M., Wong, E.E., Derian,E.L., and Babb, T. L. (1986a). Neurophysiology of limbic system pathways in the rat: projections from the subicular complex and hippocampus to the entorhinal cortex. Brain Res. 397, 205-213.

Finch, D. M., Wong, E. E., Derian, E. L., Chen, X. H., Nowlin-Finch, N. L., and Brothers, L. A. (1986b). Neurophysiology of limbic system pathways in the rat: projections from the amygdala to the entorhinal cortex. Brain Res. 370, 273-284.

Fudge, J. L., and Tucker, T. (2009). Amygdala projections to central amygdaloid nucleus subdivisions and transition zones in the primate. Neuroscience [Epub ahead of print].

Harrold, J. A., Dovey, T., Cai, X.-J., Halford, J. C. G., and Pinkney, J. (2008). Autoradiographic analysis of ghrelin receptors in the rat hypothalamus. Brain Res. 1196, 59-64.

Malik, S., McGlone, F., Bedrossian, D., and Dagher, A. (2008). Ghrelin modulates brain activity in areas that control appetitive behavior. Cell Metab. 7, 400-409.

Mayr, B., and Montminy, M. (2001). Transcriptional regulation by the phosphorylation-dependent factor CREB. Nat. Rev. Mol. Cell. Biol. 2, 599-609.

Mello, L. E., Tan, A. M., and Finch, D. M. (1992). GABAergic synaptic transmission in projections from the basal forebrain and hippocampal formation to the amygdala: an in vivo iontophoretic study. Brain Res. 587, 41-48.

Meurisse, M., Chaillou, E., and Lévy, F. (2009). Afferent and efferent connections of the cortical and medial nuclei of the amygdala in sheep. J. Chem. Neuroanat. 37, 87-97.

Moran, T.H., and Gao, S. (2006). Looking for food in all the right places? Cell Metab. 4, 233-234.

Morikawa, Y., Ueyama, E., and Senba, E. (2004). Fasting-induced activation of mitogen-activated protein kinases (ERK/p38) in the mouse hypothalamus. J. Neuroendocrinol. $16,105-112$.

O'Callaghan, J. P., and Sriram, K. (2004), Focused microwave irradiation of the brain preserves in vivo protein phosphorylation: comparison with other methods of sacrifice and analysis of multiple phosphoproteins.J. Neurosci. Methods 135, 159-168.

Ohshima, Y., Kubo, T., Koyama, R., Ueno, M., Nakagawa, M., and Yamashita, T. (2008). Regulation of axonal elongation and pathfinding from the entorhinal cortex to the dentate gyrus in the hippocampus by the chemokine stromal cell-derived factor 1 alpha. J. Neurosci. 28, 8344-8353.

Paxinos, G., and Watson, C. (1993). The Rat Brain in Stereotaxic Coordinates. San Diego, Academic Press.

Pittenger, C., Huang, Y. Y., Paletzki, R. F., Bourtchouladze, R., Scanlin, H., Vronskaya, S., and Kandel, E. R. (2002). Reversible inhibition of CREB/ATF transcription factors in region CA1 of the dorsal hippocampus disrupts hippocampus dependent spatial memory. Neuron 34, 447-462.

Shen, C. L., and Anderson, C. H. (1980). Efferents from the medial anterior hypothalamic area in the guinea pig. Brain Res. Bull. 5, 693-701.

Sheriff, S., Chance, W. T., Fisher, J. E., and Balasubramaniam, A. (1997). Neuropeptide Y treatment and food deprivation increase cyclic AMP response element-binding in rat hypothalamus. Mol. Pharmacol. 51, 597-604.

Shimizu-Albergine, M., Ippolito, D. L., and Beavo, J. A. (2001). Downregulation of fasting-induced cAMP response element-mediated gene induction by leptin in neuropeptide $\mathrm{Y}$ neurons of the arcuate nucleus. J. Neurosci. 21, 1238-1246.

Swanson, L. W. (1980). The anatomical organization of septo-hippocampal projections. Ciba Found. Symp. 58, 25-48.

Thome, J., Saki, N., Shin, K.-H., Steffen, C., Zhang, Y.-J., Impey, S., Storm, D., and Duman, R. S. (2000). cAMP response element-mediated gene transcription is upregulated by chronic antidepressant treatment. J. Neurosci. 20, 4030-4036.

Conflict of Interest Statement: The authors declare that the research was conducted in the absence of any commercial or financial relationships that could be construed as a potential conflict of interest.

Received: 10 April 2009; paper pending published: 08 May 2009; accepted: 21 May 2009; published online: 09 June 2009. Citation: Estrada NM and Isokawa M (2009) Metabolic demand stimulates CREB signaling in the limbic cortex: implication for the induction of hippocampal synaptic plasticity by intrinsic stimulus for survival. Front. Syst. Neurosci. (2009) 3:5. doi:10.3389/neuro.06.005.2009 Copyright (๑) 2009 Estrada and Isokawa. This is an open-access article subject to an exclusive license agreement between the authors and the Frontiers Research Foundation, which permits unrestricted use, distribution, and reproduction in any medium, provided the original authors and source are credited. 